\title{
The deacetylase HDAC6 is a novel critical component of stress granules involved in the stress response
}

\author{
SoHee Kwon, ${ }^{1}$ Yu Zhang, ${ }^{2}$ and Patrick Matthias ${ }^{3}$ \\ Friedrich Miescher Institute for Biomedical Research, Novartis Research Foundation, 4058 Basel, Switzerland
}

\begin{abstract}
An essential part of the cellular response to environmental stress is a reversible translational suppression, taking place in dynamic cytoplasmic structures called stress granules (SGs). We discovered that HDAC6, a cytoplasmic deacetylase that acts on tubulin and HSP90 and also binds ubiquitinated proteins with high affinity, is a novel critical SG component. We found that HDAC6 interacts with another SG protein, G3BP (Ras-GTPase-activating protein SH3 domain-binding protein 1), and localizes to SGs under all stress conditions tested. We show that pharmacological inhibition or genetic ablation of HDAC6 abolishes SG formation. Intriguingly, we found that the ubiquitin-binding domain of HDAC6 is essential and that SGs are strongly positive for ubiquitin. Moreover, disruption of microtubule arrays or impairment of motor proteins also prevents formation of SGs. These findings identify HDAC6 as a central component of the stress response, and suggest that it coordinates the formation of SGs by mediating the motor-protein-driven movement of individual SG components along microtubules.
\end{abstract}

[Keywords: HDAC6; acetylation; deacetylases; stress granules; RNA metabolism]

Supplemental material is available at http://www.genesdev.org.

Received July 3, 2007; revised version accepted October 22, 2007.

Reversible protein acetylation has emerged in recent years as one of the major forms of protein modifications whose importance has been particularly well documented in the case of the $\mathrm{N}$-terminal histone tails, and of a few transcription factors such as p53 and STAT3 (for reviews, see Kouzarides 2000; Caron et al. 2003; Glozak et al. 2005). Acetylation and deacetylation are catalyzed by histone acetylases (HATs) and histone deacetylases (HDACs). HDAC6 is a unique class II deacetylase (for reviews, see Verdin et al. 2003; Boyault et al. 2007a; Matthias et al. 2007) that contains two catalytic domains and also a C-terminal zinc finger domain (ZnF-UBP) binding with high-affinity free ubiquitin as well as mono- and polyubiquitinated proteins (Seigneurin-Berny et al. 2001; Hook et al. 2002; Boyault et al. 2006). HDAC6 is actively maintained in the cytoplasm (Verdel et al. 2000; Bertos et al. 2004), where it is found partly associated with the microtubule network. We and others have shown that HDAC6 can deacetylate tubulin as well as the microtubule network in vivo (Hubbert et al. 2002; Matsuyama et al. 2002; Zhang et al. 2003). HDAC6 associates with the chaperone-like AAA ATPase p97/VCP, a protein that is critical for proteasomal degradation of misfolded proteins. Thereby, the ratio of HDAC6 and p97/VCP modu-

Present addresses: ${ }^{1}$ The Stowers Institute for Medical Research, $1000 \mathrm{E}$. 50th St., Kansas City, MO 64110, USA; ${ }^{2}$ The CBR Institute for Biomedical Research, Harvard Medical School, Boston, MA 02115, USA.

${ }^{3}$ Corresponding author.

E-MAIL patrick.matthias@fmi.ch; FAX 41-61-697-39-76.

Article is online at http://www.genesdev.org/cgi/doi/10.1101/gad.461107. lates the levels of polyubiquitinated aggregates (Boyault et al. 2006). HDAC6 also facilitates the clearance of misfolded ubiquitinated proteins, promoting their accumulation in an aggresome, and protects cells from apoptosis following stress induced by misfolded proteins (Kawaguchi et al. 2003). At the same time, HDAC6 also controls the induction of heat-shock proteins in response to the accumulation of ubiquitinated protein aggregates (Boyault et al. 2007b). Furthermore, HDAC6 can deacetylate the chaperone Hsp90 and regulate its activity (Bali et al. 2005; Kovacs et al. 2005). Consequently, these different biochemical functions of HDAC6 impinge on diverse cellular processes. For example, HDAC6 function was found to be necessary for the formation of an immune synapse between antigen-presenting cells and $\mathrm{T}$ lymphocytes (Serrador et al. 2004) and also for nuclear translocation and transcription activation by the glucocorticoid receptor (Kovacs et al. 2005; Y. Zhang, S.H. Kwon, T. Yamaguchi, F. Cubizolles, S. Rousseau, M. Kneissel, C. Cao, N. Li, H.L. Cheng, K. Chua, et al., unpubl.). Mice lacking HDAC6 are viable despite having highly elevated tubulin acetylation in multiple organs; in addition, they exhibit a moderately impaired immune response and also a mild phenotype in the bone (Y. Zhang, S.H. Kwon, T. Yamaguchi, F. Cubizolles, S. Rousseau, M. Kneissel, C. Cao, N. Li, H.L. Cheng, K. Chua, et al., unpubl.).

One of the most immediate responses to cellular stress is a reversible block of mRNA translation, triggered by phosphorylation of the translation initiation factor eIF $2 \alpha$ 
under the action of several stress-sensing kinases such as PKR or PERK (Bertolotti et al. 2000; Williams 2001). Thereby, translationally stalled mRNAs are sequestered in dynamic cytoplasmic structures called stress granules (SGs). These granules represent a complex assembly of initiation factors, such as eIF3 or eIF4E; proteins involved in translation control, such as T-cell intracellular antigen (TIA-1) or Fragile X mental retardation protein (FMR1); proteins implicated in RNA remodeling or degradation, such as $\mathrm{HuR}$, tristetraproline (TTP), or Staufen; as well as $40 \mathrm{~S}$ ribosome subunits (for review, see Anderson and Kedersha 2006). In addition, SGs also contain various polyadenylated mRNAs whose translation has been arrested. However, mRNAs encoding stress-induced proteins, such as heatshock proteins, are excluded from SGs and are spared from translational inhibition (Kedersha and Anderson 2002; Anderson and Kedersha 2006). It is thought that SGs are sites where triage takes place in order to direct RNAs to degradation in processing bodies (PBs), or to recycle mRNAs for translation. In addition, very recent evidence suggests that parts of the microRNA pathway may also take place in SGs that contain Argonaute proteins and microRNAs such as let-7 (Leung et al. 2006).

Here, we report the identification of G3BP-1 (RasGTPase-activating protein SH3 domain-binding protein 1, hereafter G3BP), an SG component, as a novel protein interacting with HDAC6 in vivo and in vitro. This protein is conserved between species, and orthologs are found in Drosophila, humans, and mice. G3BP has been implicated in modulating Ras activity and the cell cycle, by binding to the RasGAP protein (Pazman et al. 2000; Kennedy et al. 2001). The precise function of G3BP is not understood yet, but it appears to be essential in the mouse where inactivation of the G3BP gene leads to embryonic lethality and growth retardation (Zekri et al. 2005). G3BP has attracted attention recently as it was found to increase decay of the c-Myc RNA and to localize to SGs (Tourriere et al. 2001, 2003). We show that HDAC6 is recruited to SGs and that pharmacological HDAC inhibition leads to impaired SG assembly. Indeed, HDAC6-deficient mouse embryo fibroblasts (MEFs) fail to form SGs, although they exhibit normal phosphorylation of eIF2 $\alpha$ in response to stress. Furthermore, inactivating mutations in the catalytic domains or in the ZnF-UBP domain of HDAC6 impair SG assembly. Moreover, SG formation is abolished by disruption of microtubule arrays or by impairment of dynein motor proteins. We also found that HDAC6 is required for the cells to recover from oxidative stress: In the absence of intact HDAC6 function, cells that have been treated with arsenite undergo apoptosis. Based on these results, we propose that HDAC6 is a central component of the stress response, regulating SG formation and potentially contributing to the control of RNA metabolism and translation.

\section{Results}

HDAC6 interacts with G3BP-1 in vivo and in vitro

To identify novel proteins associating with HDAC6, we established a stable cell line expressing tagged HDAC6
(S-HDAC6-293) and used it for immunoprecipitations. Coprecipitating proteins were separated by SDS-PAGE and analyzed by mass spectrometry. By this approach, we identified G3BP as a prominent HDAC6-interacting partner. To verify the interaction between G3BP and HDAC6, coimmunoprecipitation and pull-down assays were established. As shown in Figure 1A, when HEK $293 \mathrm{~T}$ cells were transiently cotransfected with constructs encoding epitope-tagged G3BP and HDAC6, the two proteins were found to efficiently coprecipitate, irrespective of the order. Interaction could also be evidenced in an in vitro binding assay, using a bacterially expressed GST-G3BP fusion protein and extracts from HDAC6-transfected HEK $293 \mathrm{~T}$ cells or in vitro translated HDAC6 protein (Fig. 1B; data not shown). Importantly, interaction between G3BP and HDAC6 could also be demonstrated with endogenous proteins from $293 \mathrm{~T}$ cells, as shown in Figure 1C. The interaction is specific for HDAC6, as other HDACs, such as HDAC1 or HDAC4, fail to coimmunoprecipitate with G3BP (Fig. 1D). Taken together, these results indicate that G3BP is a bona fide novel specific interaction partner of the deacetylase HDAC6.

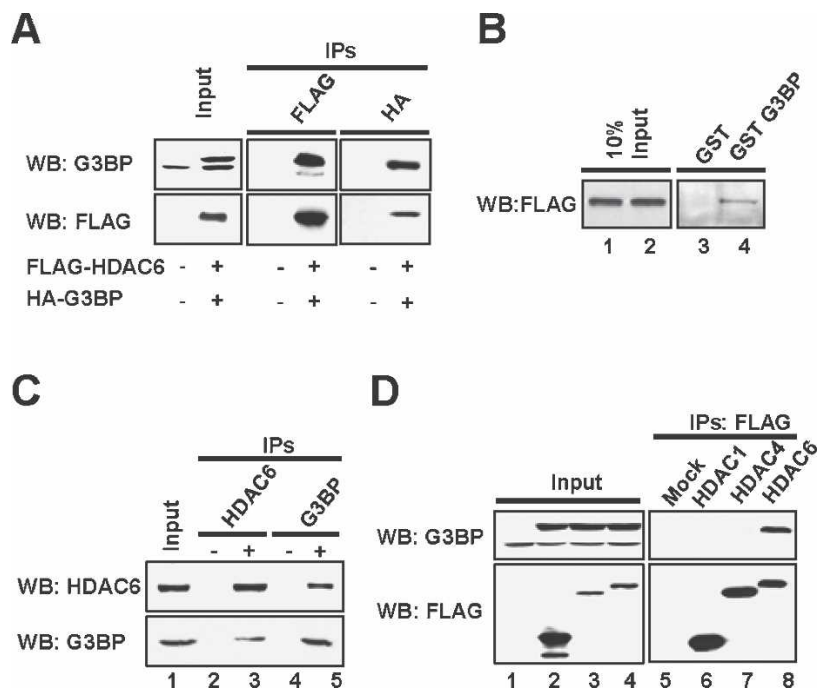

Figure 1. HDAC6 associates with G3BP. (A) Coimmunoprecipitation assay. 293T cells were cotransfected with an empty expression vector, Flag-tagged HDAC6, or HA-tagged G3BP. Interaction was measured by immunoprecipitation with an antiHA or anti-Flag antibody, followed by immunoblotting with an antibody to detect G3BP or HDAC6, as indicated. Ten percent of total cell lysates used in immunoprecipitation are shown as input. (B) GST pull-down assay. Equal amounts of extracts from 293 T cells transiently transfected with a vector encoding FlagHDAC6 were incubated with beads loaded with GST alone or a GST-G3BP fusion protein. After washing, bound proteins were run on SDS-PAGE, and retained HDAC6 was detected by Western blotting using an anti-Flag antibody. $(C)$ Coimmunoprecipitation assay with endogenous proteins. 293T cell extracts were immunoprecipitated with an anti-HDAC6 or anti-G3BP antibody, followed by immunoblotting with the indicated antibodies. (D) Extracts from 293T cells transfected with Flag-tagged HDACs and GFP-tagged G3BP were immunoprecipitated with an anti-Flag antibody and immunoblotted for G3BP. 
Phosphorylation of G3BP modulates the interaction with HDAC6

To define the domain(s) required for interaction between HDAC6 and G3BP, truncated proteins were expressed by transfection in $293 \mathrm{~T}$ cells, and interaction was tested by coimmunoprecipitation assays. As shown in Figure 2, A and $\mathrm{B}$, the full-length HDAC6 protein as well as deletion mutants (Seigneurin-Berny et al. 2001) containing at least one HDAC domain coimmunoprecipitated efficiently with G3BP. The $\mathrm{N}$-terminal region (first 84 amino acids) of HDAC6 bound weakly to G3BP, whereas the C-terminal last 324 amino acids did not bind appreciably (Fig. 2B, lanes 4,6). In addition, we tested HDAC6 proteins with point mutations in the catalytic site of either or both of the HDAC domains (Grozinger et al. 1999). Both the single and double mutants could be coprecipitated with G3BP as efficiently as the wild-type enzyme (Fig. 2D). Thus, the interaction between HDAC6 and G3BP is mediated by either HDAC domain, but does not depend on an intact catalytic center. Next, a series of G3BP deletion constructs was tested in a similar man-
A

B

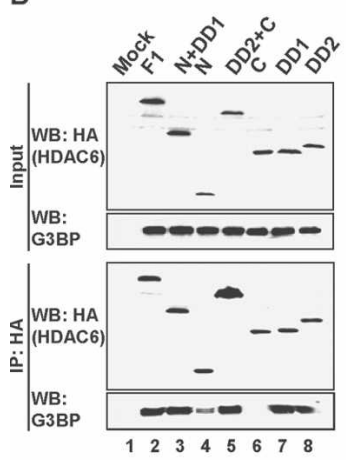

C

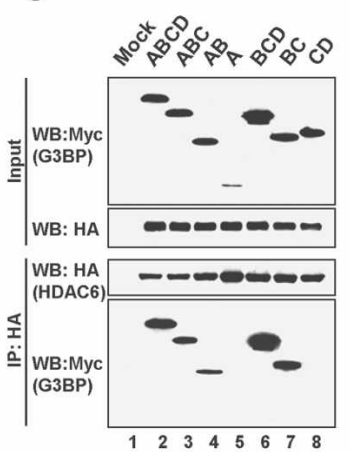

D

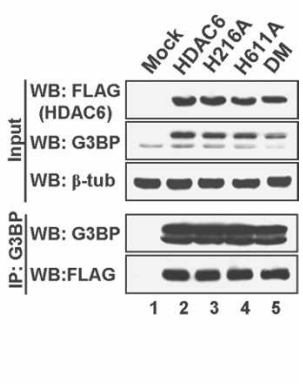

E

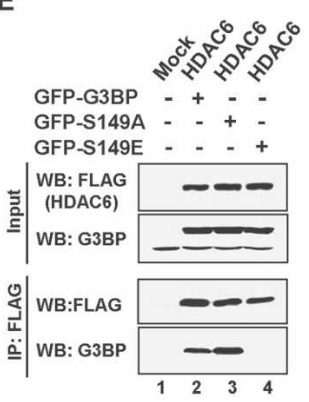

$F$

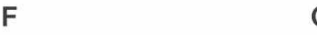

G Oka - . + - -

Van - - -+

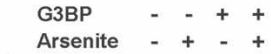

I

Arsenite -++

횔ㅇ $\begin{aligned} & \text { WB: HDAC6 } \\ & \text { WB: G3BP }\end{aligned}$

兽WB: HDAC6

: WB: G3BP

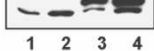

$\lambda$ PPase - - - - +

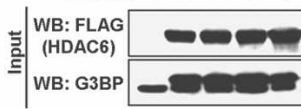

WB:FLAG

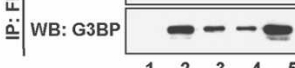

H

Van - + - -

$\lambda$ PPase - - +

Ars $\quad-\cdots+$

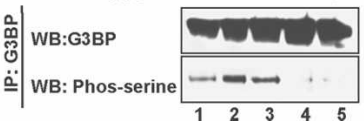

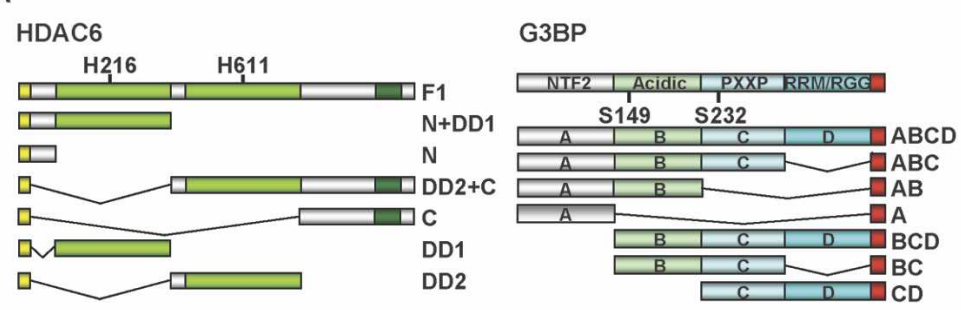

Oka $\quad+\quad+-\cdot$

Figure 2. HDAC-6 interacts with G3BP via the HDAC domains, and phosphorylation of G3BP modulates its interaction with HDAC6. (A) Schematic representation of the $\mathrm{N}$-terminally HA-tagged HDAC6 deletion constructs and C-terminally Myctagged G3BP truncated mutants used in this study. The two G3BP serine residues that can be phosphorylated are indicated. $(B-G)$ Coimmunoprecipitation assays. One representative experiment is presented $(n=3-4)$. (B) HDAC6 interacts with G3BP through the HDAC domains. (Lanes 2-8) 293T cells were cotransfected with the indicated HDAC6 expression vectors together with a G3BP full-length expression vector, and cellular extracts were prepared. Expression of HDAC6 or G3BP was measured by Western blot with an anti-HA or anti-G3BP antibody. Association with HDAC6 was measured by performing an immunoprecipitation with an antiHA antibody, followed by analysis of the precipitate by Western blotting with the anti-G3BP antibody. (C) G3BP interacts with HDAC6 through the acidic $\mathrm{B}$ domain. The domain in G3BP required to interact with HDAC6 was identified by testing extracts from cells transfected with full-length HDAC6 and deletion mutants of G3BP. Analysis was done as in $B .(D)$ The catalytic domains of HDAC6 are not critical for interaction with G3BP. Extracts from 293T cells cotransfected with HA-tagged G3BP and the indicated Flag-tagged HDAC6 point mutants were immunoprecipitated with an anti-G3BP antibody. In the HDAC6 mutant proteins, the histidine at position 216 or 611 was mutated to alanine (H216A, H611A). (DM) Double mutant protein. (E) G3BP phosphorylation at Ser149 modulates interaction with HDAC6. GFP-fused wild-type or point mutants G3BP and Flag-tagged HDAC6 were cotransfected into $293 \mathrm{~T}$ cells, and lysates were analyzed by immunoprecipitation and Western blotting. The lysates were immunoprecipitated with an anti-Flag antibody, followed by immunoblotting with an anti-Flag antibody, and were then reblotted for G3BP. Ten percent of total cell lysates used in immunoprecipitation are shown as input. $(F)$ Arsenite-induced G3BP dephosphorylation promotes interaction with HDAC6. (Lanes 2,4) 293 T cells were mock-transfected or transfected with HA-tagged G3BP and were treated with $1 \mathrm{mM}$ arsenite for
$1 \mathrm{~h}$ prior to lysis; extracts were then used for coimmunoprecipitation with an anti-G3BP antibody and analysis by immunoblotting, as indicated. $(G)$ Phosphatase inhibition reduces the interaction between G3BP and HDAC6. 293T cells were cotransfected with HA-tagged G3BP and Flag-tagged HDAC6 (lanes 2-5) and treated with phosphatase inhibitors (lanes 3,4); alternatively, cell lysates were incubated in vitro with $\lambda$ phosphatase (lane 5). Subsequent analysis was carried out as in E. (H) 293T cells (lanes 1-3,5) or extracts thereof (lane 4) were treated as indicated, and extracts were prepared and immunoprecipitated with an anti-G3BP antibody. The precipitate was then immunoblotted with anti-G3BP antibody and with an anti-phosphoserine antibody. (Ars) Arsenite; (Oka) okadaic acid; (Van) orthovanadate; $\lambda$ Ppase) $\lambda$ phosphatase. 
ner. As shown in Figure 2C, the acidic-rich domain of G3BP (labeled B in the schematic), containing the serumdependent phosphorylation site (see below), is required for binding to HDAC6, whereas the N-terminal NTF2like domain (labeled A) and the C-terminal half of the protein (labeled CD) are dispensable.

G3BP can be phosphorylated at Ser149 and Ser232 (Fig. 2A; Tourriere et al. 2001), and phosphorylation at Ser149 has been shown to dominantly inhibit SG formation (Tourriere et al. 2003). Furthermore, arsenite or heatshock treatment of cells, as well as H-ras activation, induce dephosphorylation of G3BP at S149, which can then assemble in SGs (Gallouzi et al. 1998; Tourriere et al. 2001, 2003). To test whether the phosphorylation of G3BP at Ser149 influences its interaction with HDAC6, we transiently cotransfected constructs encoding wildtype or phosphomutant G3BP proteins and Flag-tagged HDACs into HEK 293 T cells and performed coimmunoprecipitation assays. Both the wild-type and the S149A nonphosphorylable G3BP mutant coprecipitated with HDAC6; however, the phosphorylation-defective mutant coprecipitated more robustly than the wild-type (Fig. 2E). In contrast, the phosphomimetic G3BP S149E mutant completely failed to coprecipitate, although it was expressed at equivalent levels to the other proteins. In agreement with this finding, dephosphorylation of G3BP, induced by treatment of cells with arsenite or incubation of extracts with $\lambda$ phosphatase, led to increased interaction with HDAC6 (Fig. 2F [lanes 2,4], G [lane 5]). Moreover, the interaction was weaker when phosphorylation of G3BP was increased by treatment of the cells with a phosphatase inhibitor such as okadaic acid or vanadate (Fig. 2G, lanes 3,4). In agreement with this, treatment of the cells with phosphatase inhibitors prior to extract preparation led to increased phosphorylation of G3BP (Fig. 2H, lanes 2,3); conversely, G3BP phosphorylation was reduced upon arsenite treatment of the cells (Fig. 2H, lane 5) or in vitro treatment of the extract with $\lambda$ phosphatase (Fig. $2 \mathrm{H}$, lane 4). Together, these results therefore demonstrate that the phosphorylation status of G3BP modulates its interaction with HDAC6.

\section{HDAC6 and G3BP colocalize and are recruited to SGs}

The biochemical interaction between HDAC6 and G3BP and its regulation by phosphorylation prompted us to test whether these two proteins colocalize in the cell. In exponentially growing HeLa cells, both proteins are diffusely distributed throughout the cytoplasm, as had been observed previously (Fig. 3; Gallouzi et al. 1998; Zhang et al. 2003). It has been recently reported that G3BP is recruited to SGs in cells exposed to stress (Tourriere et al. 2003). To test whether HDAC6 is also recruited to SGs, we examined the localization of endogenous HDAC6 or G3BP, following treatment of the cells with arsenite, a strong inducer of oxidative stress. As shown in Figure 3, under these conditions G3BP and HDAC6 were both found in small cytoplasmic foci, and the merged picture shows good colocalization of the two proteins. To con- firm this result, arsenite-treated cells were also stained for TIA-1, another marker of SGs. In this case as well, endogenous HDAC6 was concentrated at discrete cytoplasmic foci and colocalized with TIA-1. The perfect colocalization of HDAC6 with G3BP or TIA-1 therefore indicates that HDAC6 is a novel component of SGs. Previous studies have shown that the components of SGs vary with the stimulus used to elicit their assembly: Heat-shock-induced SGs contain HSP27, whereas arsenite-induced SGs do not (Kedersha et al. 1999), and SGs containing G3BP or TIA-1 are only partially overlapping (Tourriere et al. 2003). To address whether the recruitment of HDAC6 into SGs is restricted to a specific form of stress, HeLa cells were treated with different SG-inducing stimuli such as arsenite, UV irradiation, CCCP (mitochondrial stress), or heat shock and were immunostained with antibodies against G3BP or HDAC6. As shown in Figure 3, G3BP localized to SGs under all conditions tested; remarkably, HDAC6 was also found in SGs irrespective of the induction stimulus. When the stress stimulus is removed and cells are returned to normal culture conditions, SGs disassemble rapidly (Anderson and Kedersha 2002; Kedersha et al. 2002). We therefore examined the kinetics of SGs assembly and disassembly, using G3BP and HDAC6 as markers. We found that both proteins behaved identically also under these conditions (Supplementary Fig. S1). These results altogether indicate that HDAC6 and G3BP are recruited to the same SGs in response to stress, with identical kinetics, and that HDAC6 is an integral (stable) component of SGs.

\section{The deacetylase activity of HDAC6 can regulate $S G$ formation}

To identify the functional domain(s) of HDAC6 that direct the protein to SGs, expression vectors encoding tagged HDAC6 deletion mutants and G3BP were transiently cotransfected into HeLa cells, and the subcellular localization of these proteins was determined under control or oxidative stress conditions. The results are summarized and representative photomicrographs are presented in Supplementary Figure S2. All of the HDAC6 deletion mutants were localized in the cytoplasm, in agreement with our earlier results. Consistent with the immunoprecipitation results defining the interaction with G3BP, the full-length HDAC6 and deletion mutants containing at least one HDAC domain were recruited to SGs; in contrast, mutants containing only the $\mathrm{N}$ - or the C-terminal portion of HDAC6 failed to localize to SGs. Thus, either of the two HDAC domains is necessary and sufficient to direct HDAC6 to SGs.

To examine whether the deacetylase activity of HDAC6 is required for SG formation, we first treated HeLa cells with the HDAC inhibitor, TSA, which inhibits all known HDACs, or with butyrate, which inhibits HDACs other than HDAC6 (Guardiola and Yao 2002). As shown in Figure 4, cells treated with TSA and arsenite exhibited a hyperacetylated microtubule network and formed fewer or no HDAC6-positive SGs. In con- 
trast, treatment of the cells with butyrate did not prevent SG formation, suggesting that the deacetylase activity of HDAC6 might be important for formation of these structures.

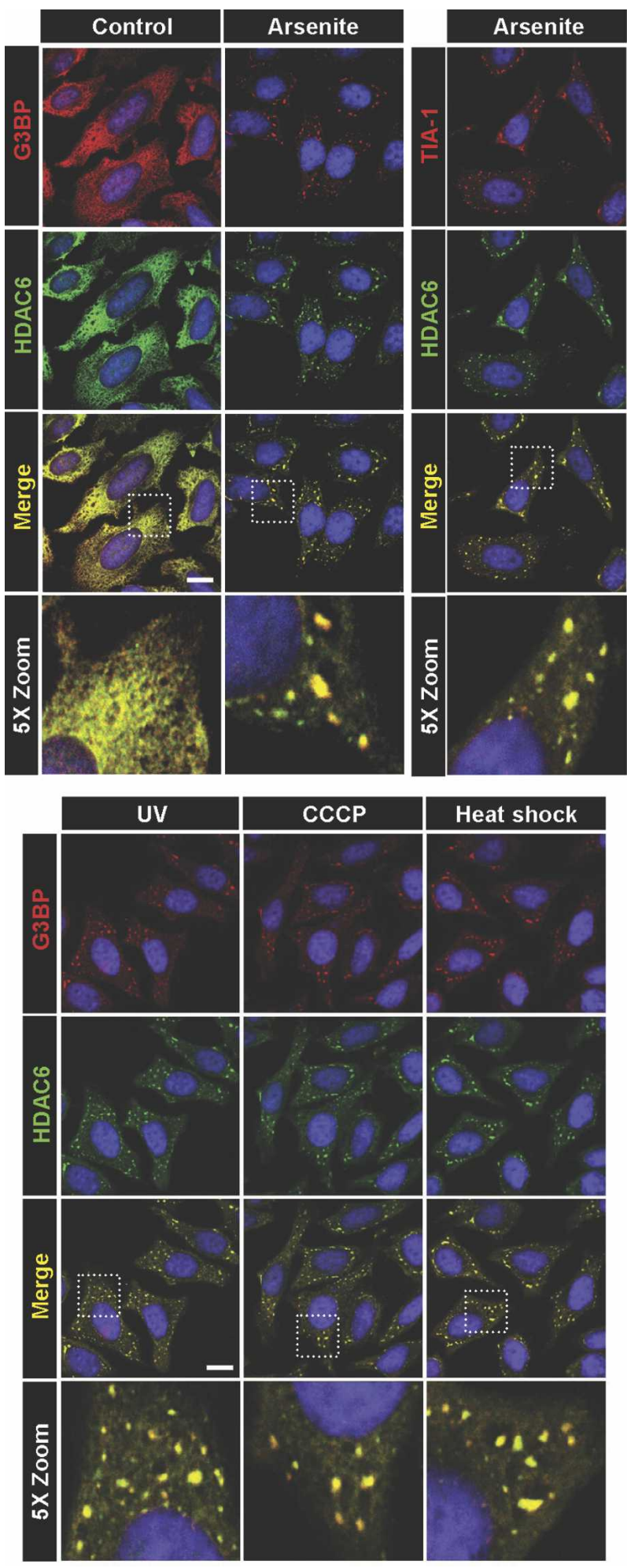

\section{HDAC6-deficient MEFs exhibit impaired ability to form SGs}

To examine the importance of HDAC6 in the regulation of SG assembly, we used wild-type or HDAC6 knockout (KO) MEFs to test their capacity to form SGs following stress. Whereas wild-type MEFs readily assembled SGs in response to arsenite treatment, HDAC6 KO MEFs failed to exhibit morphologically discrete SGs, as determined by using antibodies against two independent markers of SGs, TIA-1 and G3BP (Fig. 5A,B). The same results were obtained using other stress forms such as heat shock, UV, or CCCP (Supplementary Fig. S3). We also quantified the percentage of cells forming SGs and the kinetics of their assembly. As shown in Figure 5B, in wild-type MEFs up to $\sim 80 \%$ of the cells were positive for SGs after $1 \mathrm{~h}$ of arsenite treatment, as in HeLa cells (data not shown); in contrast, in HDAC6-deficient cells, no more than $\sim 20 \%-25 \%$ of the cells were ever positive. Furthermore, while wild-type cells exhibited SGs already after 5-10 min of arsenite treatment, and reached a plateau after $30 \mathrm{~min}$, HDAC6-deficient cells showed a much-delayed response (Fig. 5C).

One of the earliest steps in the formation of SGs is the phosphorylation of eIF2 $\alpha$ by stress-activated kinases, such as PKR or PERK, which leads to inhibition of translation initiation (Kedersha et al. 1999, 2002; Williams 2001). We therefore tested whether loss of HDAC6 influences eIF $2 \alpha$ phosphorylation in response to stress. As presented in Figure 5D, both wild-type (lanes 2-4) and HDAC6 KO MEFs (lanes 6-8) exhibited a similar increase in phospho-eIF2 $\alpha$ upon arsenite treatment. We also examined the kinetics of eIF2 $\alpha$ phosphorylation by treating cells with arsenite and analyzing extracts at different time points thereafter. As shown in Figure 5E, cells of both genotypes showed a comparably rapid appearance of phospho-eIF $2 \alpha$, indicating that impaired eIF $2 \alpha$ phosphorylation cannot explain the failure of HDAC6 knockout cells to efficiently assemble SGs. In agreement with the above observations, we also found that global translation was not altered in the absence of HDAC6, as polysomal profiles were identical in wildtype or $H D A C 6^{-/ y}$ MEFs (Supplementary Fig. S4). Thus, the effect of HDAC6 on SG formation is downstream from eIF $2 \alpha$ phosphorylation and protein translation arrest.

Figure 3. HDAC6 localizes to SGs. Exponentially growing HeLa cells were control-treated or stressed by exposure to $1 \mathrm{mM}$ arsenite for $1 \mathrm{~h}, 100 \mathrm{~mJ}$ of UV irradiation, $1 \mu \mathrm{M}$ CCCP for 90 $\min$, or heat $\left(44^{\circ} \mathrm{C}\right)$ for $1 \mathrm{~h}$. Subsequently, cells were fixed and stained for G3BP, HDAC6, or TIA-1. Double-immunofluorescence experiments were performed using anti-HDAC6 and antiG3BP or anti-TIA-1/TIAR antibody and labeled secondary antibodies (Alexa Fluor 488 [green], and Alexa Fluor 594 [red]). Nuclei were counterstained using DAPI (blue). Localization of proteins was monitored by confocal microscopy. Yellow represents colocalization. Enlargements of boxed regions are shown in the bottom row. Bar, $10 \mu \mathrm{m}$. 


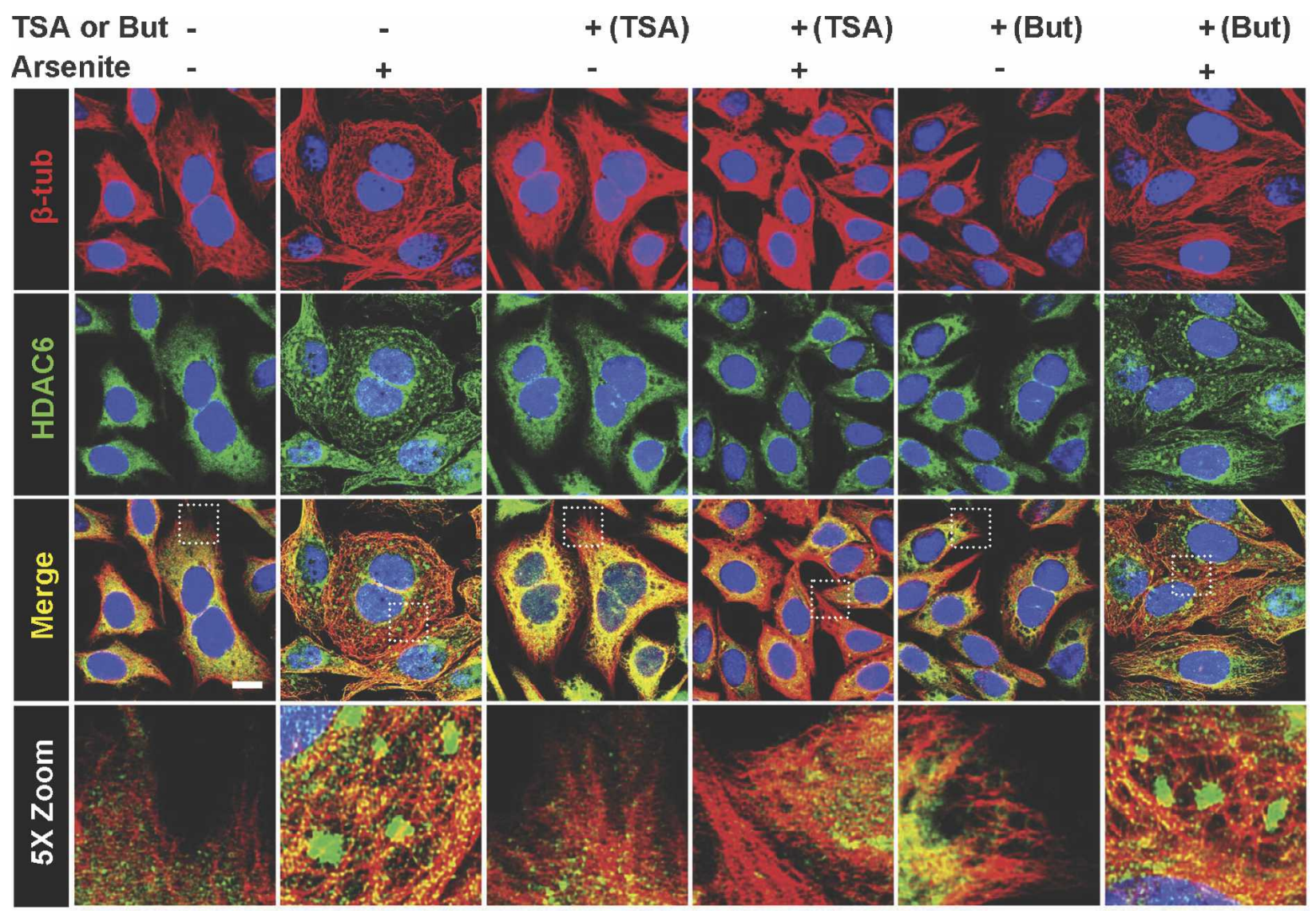

Figure 4. The deacetylase activity of HDAC6 is critical for assembly of SGs. HDAC inhibition impairs formation of SGs. HeLa cells were control-treated or treated with $500 \mathrm{nM}$ TSA or $5 \mathrm{mM}$ Butyrate for $4 \mathrm{~h}$ prior to treatment with arsenite for $1 \mathrm{~h}$ and fixation. Double-immunostaining experiments were carried out with anti-Ac- $\alpha$-tubulin and anti-HDAC6 antibodies, and analysis was done by confocal microscopy. Enlargements of boxed regions are shown in the bottom row. Bar, $10 \mu \mathrm{m}$.

\section{Intact HDAC6 function is required for SG formation}

To better define the role of HDAC6 in controlling SG formation, we made use of $H D A C 6^{-/ y}$ MEFs in which wild-type or mutant forms of this enzyme have been re-expressed by retroviral transduction. As seen in Figure $5 \mathrm{~F}$, these cells all express wild-type levels of HDAC6 and behave as expected with respect to tubulin acetylation: The cells expressing wild-type HDAC6 have a tubulin acetylation level similar to wild-type MEFs (Fig. 5F, lane 3), while the cells expressing a deacetylase mutant form of HDAC6 have hyperacetylated tubulin (Fig. 5F, lane 4), just like $H D A C 6^{-/ y}$ MEFs. In addition, cells expressing an HDAC6 protein that cannot bind ubiquitin owing to a mutation in the ZnF-UBP domain also have a wild-type level of tubulin acetylation (Fig. 5F, lane 5). These different cell lines, as well as wild-type control MEFs, were used to examine SG formation following stress induction. As shown in Figure 5G, expression of HDAC6 in $H D A C 6^{-/ y}$ MEFs largely restored SG formation; in contrast, expression of a deacetylase mutant HDAC6 failed to do so. Moreover, cells expressing the non-ubiquitinbinding HDAC6 mutant also appeared to lack SGs. To further solidify these results, we quantified in the differ- ent cell lines both the percentage of cells positive for SGs, as well as the number of large $(>1.0 \mu \mathrm{m}$, see legend) SGs per cell. From the data presented in Figure $5 \mathrm{H}$, it is clear that the deacetylase activity as well as the ubiquitin-binding activity of HDAC6 are both necessary for promoting SG assembly. In fact, the cells expressing the non-ubiquitin-binding mutant HDAC6 are more severely impaired than the $H D A C 6^{-/ y} \mathrm{MEFs}$, indicating that the mutant protein may act as a dominant negative. Because of this observation, we tested whether ubiquitin is present in SGs. As shown in Supplementary Figure S5, in untreated HeLa cells, immunostaining for ubiquitin shows a diffuse pattern in the cytoplasm as well as in the nucleus. In contrast, arsenite treatment of the cells leads to the appearance of a punctate staining in the cytoplasm that perfectly colocalizes with HDAC6 (or other SG markers) (data not shown). In addition, the same results have been obtained in MEFs; we therefore conclude that SGs contain ubiquitinated proteins and that staining for ubiquitin can be used to monitor the presence of these structures. Remarkably, we also observed that shortterm (3-h) inhibition of the ubiquitin-proteasome system with MG132 leads to the appearance of SGs that are positive for ubiquitin, HDAC6, and other markers. In 
A

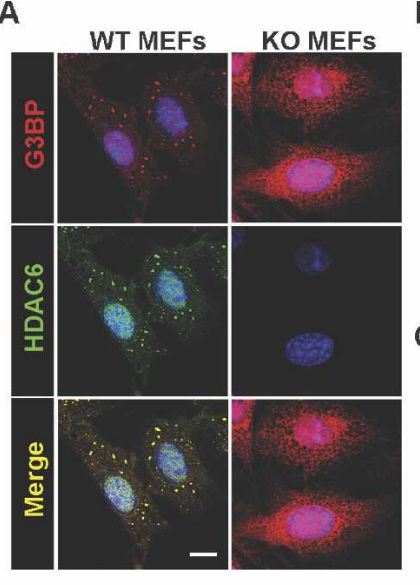

B

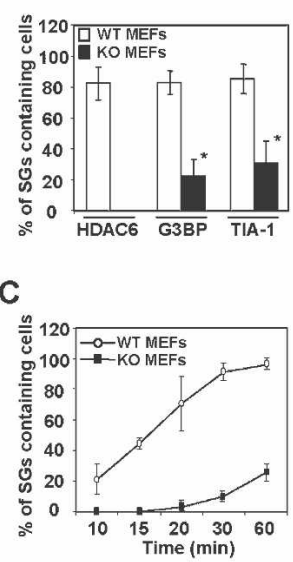

H

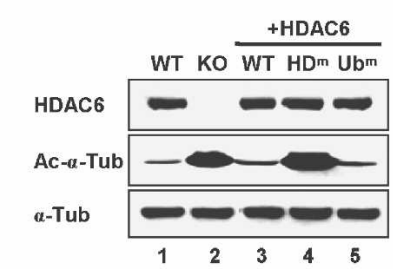

G

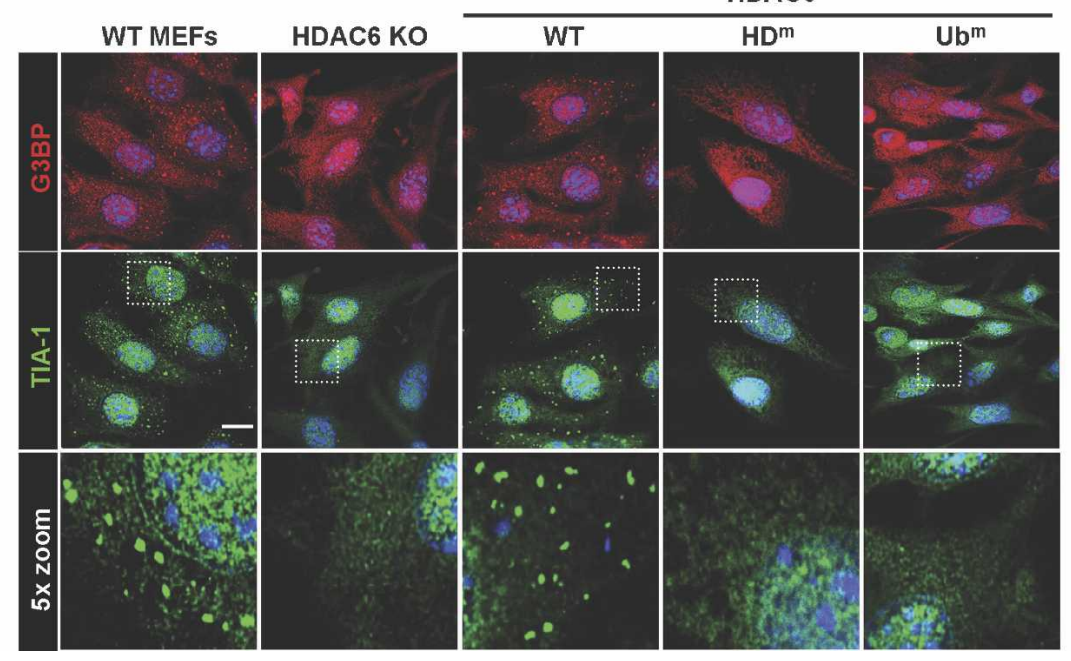

D

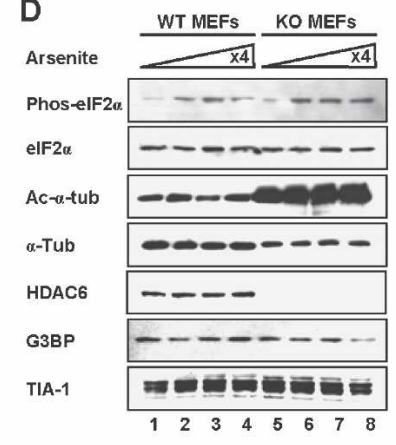

E

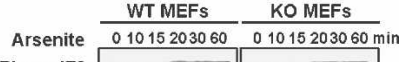
PhoselF2a. --elF2 $\alpha$. HDAC6
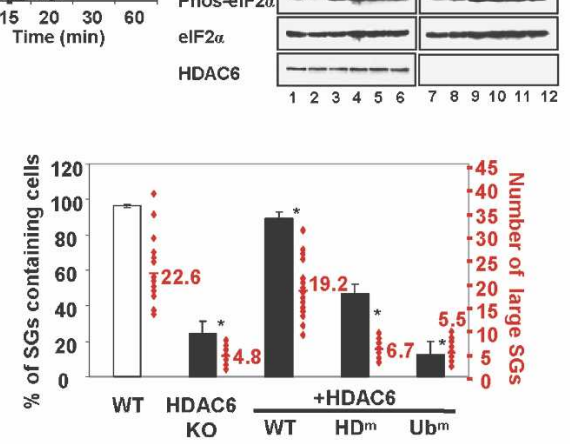
KO WT HD Ub $^{m}$ +HDAC6 
the arsenite-induced stress and showed no apoptosis up to $48 \mathrm{~h}$. In stark contrast, the mutant MEFs were all dramatically impaired in their capacity to recover from the stress. In this case, at $12 \mathrm{~h}$ post-stress, $\sim 10 \%-15 \%$ of the cells were apoptotic, and at $48 \mathrm{~h}$ this percentage increased to $\sim 45 \%-55 \%$. Thus, intact HDAC6 function is essential for normal cellular recovery from arsenite-induced oxidative stress.

SG formation depends on the microtubule system and is mediated by motor protein function

Formation of SGs is very rapid, and within minutes microscopically visible structures assemble in wild-type cells (Fig. 5C; Kedersha et al. 2000, 2005). We showed here that HDAC6 is a critical factor for SG assembly, yet it is not known what other mechanisms are involved. We therefore investigated the importance of the cytoskeleton in SG formation by testing the effect of drugs that alter the microtubules or the actin network. We found, in agreement with the work of Ivanov et al. (2003), that treatment of HeLa cells with the microtubule network destabilizing drug nocodazole dramatically reduced the appearance of SGs following induction by arsenite (Fig. 6A,B). In this case, $<20 \%$ of the cells contained SGs (Fig. 6B), which were of very small size. In line with this, another microtubule-disrupting drug, colchicine, also prevented formation of SGs /data not shown). In contrast, stabilization of the microtubules network with taxol did not impair the formation of SGs (data not shown). Thus, microtubules provide a necessary scaffold for the assembly of SG components. Moreover, disruption of the actin network by latrunculin B or cytochalasin D did not impair formation of SGs even though it was associated with significant contraction of the cell body (Supplementary Fig. S7).

HDAC6 was shown previously to interact with microtubules and also with dynein, thereby facilitating the accumulation of misfolded proteins into an aggresome (Hubbert et al. 2002; Kawaguchi et al. 2003; Zhang et al. 2003). We thus wondered whether motor proteins could also be involved in the formation of SGs. To test this hypothesis, we treated cells with two inhibitors of dynein ATPase function, EHNA or vanadate, in conjunction with arsenite. In both cases, the microtubule network remained intact, and the localization of HDAC6 was not impaired; however, cells that were arsenitetreated in the presence of these inhibitors did not exhibit SGs (Fig. 6A,B). Thus, dynein motor proteins are required, in conjunction with HDAC6, to assemble SGs along the microtubules. In addition, we also tested whether SG components might interact with microtubules. For this, 293T whole-cell extracts were prepared, tubulin was immunoprecipitated, and the presence of SG proteins in the precipitate was detected by immunoblotting. As shown in Figure 6C, while G3BP did not coprecipitate appreciably, both TIA-1 and eIF3 did. Interestingly, treatment of the cells with arsenite prior to extract preparation did not change the level of association.

\section{HDAC6 does not influence assembly of PBs}

It has been shown recently that PBs, where mRNA storage and degradation take place (van Dijk et al. 2002; Sheth and Parker 2003; Pillai et al. 2005; Teixeira et al. 2005), are dynamically linked to SGs (Kedersha et al. 2005) and often found in close juxtaposition. Because of this, we examined whether HDAC6 also colocalizes with decapping enzyme 1 (Dcpla), a marker for PBs. As shown in Figure 7A, PBs are well visible in controltreated HeLa cells, as well as in arsenite-treated cells, where their number and size increase slightly, in agreement with previous results (Kedersha et al. 2005; Teixeira et al. 2005). Furthermore, many PBs are indeed found next to SGs, evidenced here by HDAC6 staining, but the same results are obtained when SGs are detected by G3BP or TIA-1 staining (data not shown). Remarkably, no colocalization of HDAC6 to the PBs was observed. We next examined formation of PBs in wild-type MEFs or in MEFs lacking HDAC6: Arsenite-treated wildtype MEFs exhibit numerous PBs, many of which are next to HDAC6- or G3BP-positive SGs (Fig. 7B). Strikingly, in HDAC6 knockout MEFs, G3BP fails to form SGs, as shown above, but PBs are present in normal number and size. Thus, we conclude that HDAC6 is not required for the formation of $\mathrm{PBs}$ and that they can form independently of SGs.

\section{Discussion}

The cellular response to stress entails a very rapid reversible suppression of mRNA translation accompanied by relocalization of mRNPs into discrete cytoplasmic SGs, where they are remodeled and protected from degradation (for review, see Anderson and Kedersha 2006). Here we show that the deacetylase HDAC6 is a critical factor for SG assembly. Our results demonstrate that HDAC6 regulates SG formation by integrating different cellular processes, all of which we show to be important: protein acetylation, ubiquitination, integrity of the microtubule network, and function of motor proteins. Furthermore, we show that cells lacking intact HDAC6 function fail to recover properly from arsenite-induced stress and undergo apoptosis.

\section{G3BP is a novel interacting partner of HDAC6 in vivo}

We identified the SG-associated protein G3BP as a novel interacting partner of HDAC6. We found that the HDAC domains of HDAC6 are necessary and sufficient for binding to a central domain of G3BP rich in acidic residues; this interaction may be direct or indirect. Phosphorylation of G3BP at Ser149 has been shown to play a key role in regulating its subcellular localization, endoribonuclease activity, and also its capacity to self-aggregate and localize to SGs (for review, see Irvine et al. 2004). We showed that phosphorylation of G3BP at this residue critically regulates its interaction with HDAC6, as only nonphosphorylated G3BP can associate with HDAC6. We propose that the interaction with HDAC6 is critical 
A
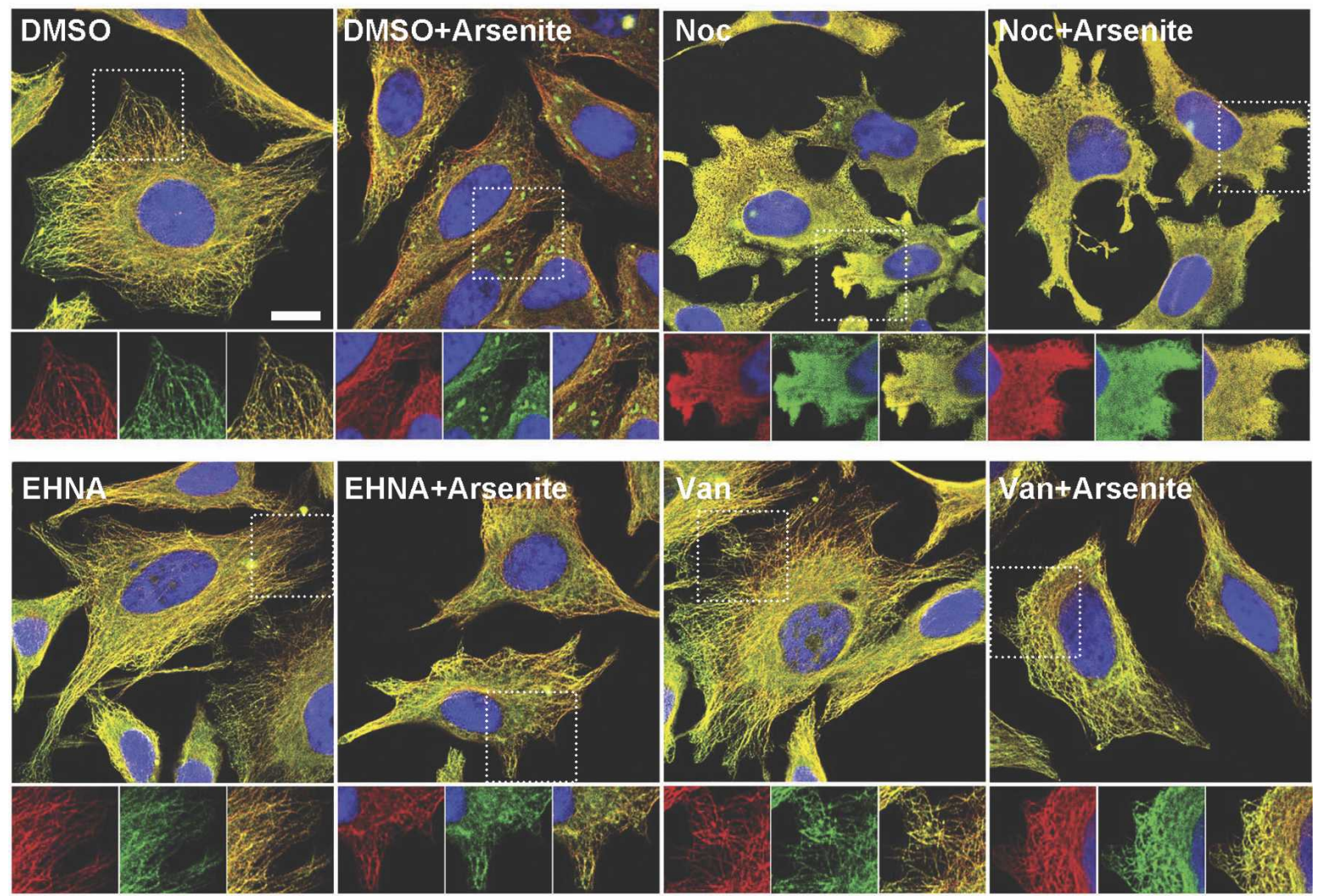

B

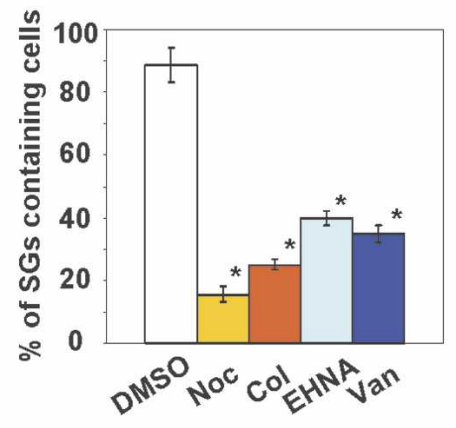

C

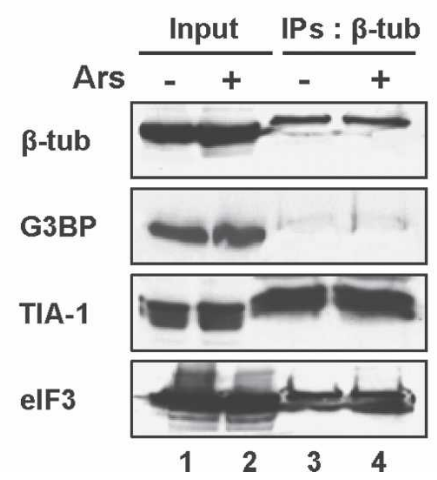

Figure 6. An intact microtubule network and dynein function are required for SG assembly. $(A)$ Hela cells were treated with $0.1 \%$ DMSO (control) for $4 \mathrm{~h}, 6.6 \mu \mathrm{M}$ Nocodazole (Noc) for $2 \mathrm{~h}, 1 \mathrm{mM}$ EHNA for $1 \mathrm{~h}$, or $0.5 \mathrm{mM}$ vanadate (Van) for $4 \mathrm{~h}$, prior to treatment for $30 \mathrm{~min}$ with arsenite, as indicated. Cells were fixed and immunostained for $\beta$-tubulin and HDAC6. The insert presents a higher magnification showing SGs on the microtubule network. (B) Quantification of the percentage of HeLa cells containing SGs under conditions of microtubule disruption or inhibition of dynein ATPase activity. The occurrence of SGs was estimated based on HDAC6 immunostaining. Error bars represent the SD calculated from 200 cells in nine random fields. Student's $t$-test was used for statistical analysis. $\left(^{*}\right) P<0.01$ versus control. (Noc) Nocodazole; (Col) Colchicine; (EHNA) erythro-9-[3-(2-Hydroxynonyl)]adenine; (Van) Orthovanadate. Bar, $10 \mu \mathrm{m}$. (C) SG components interact with $\beta$-tubulin. 293T cells were treated without or with $1 \mathrm{mM}$ arsenite for $1 \mathrm{~h}$. Cell lysates were immunoprecipitated with an anti- $\beta$-tubulin antibody, and the precipitated material was immunoblotted with the indicated antibodies. Ten percent of total cell lysates used in immunoprecipitations are shown as input. (Ars) Arsenite.

for the localization of G3BP in SGs. In the case of TTP, another SG-associated RNA-destabilizing factor, arsenite-induced phosphorylation promotes rapid exit from SGs and concurrently inhibits its ability to promote ARE-mediated mRNA decay (Stoecklin et al. 2004).
HDAC6 is a novel critical component of SGs, but not of $P B S$

We observed that, under normal conditions, both HDAC6 and G3BP are predominantly cytoplasmic pro- 
Kwon et al.

A

B
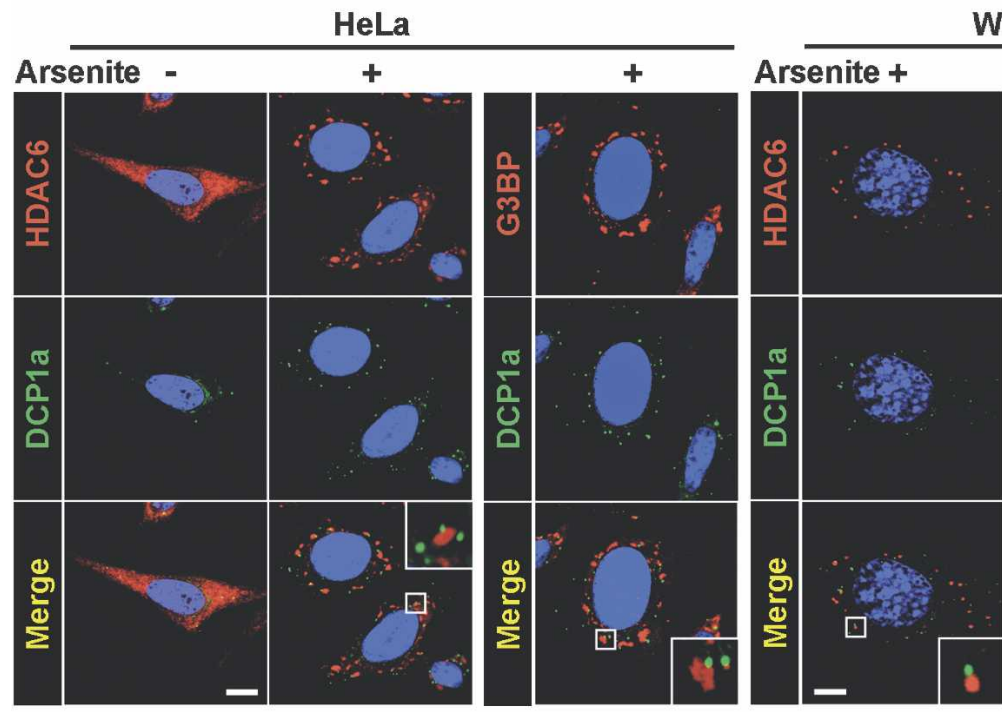

WT MEFs

KO MEFs
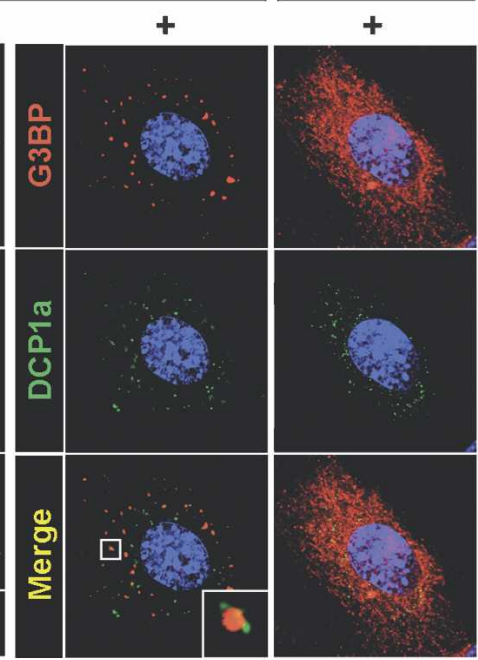

C

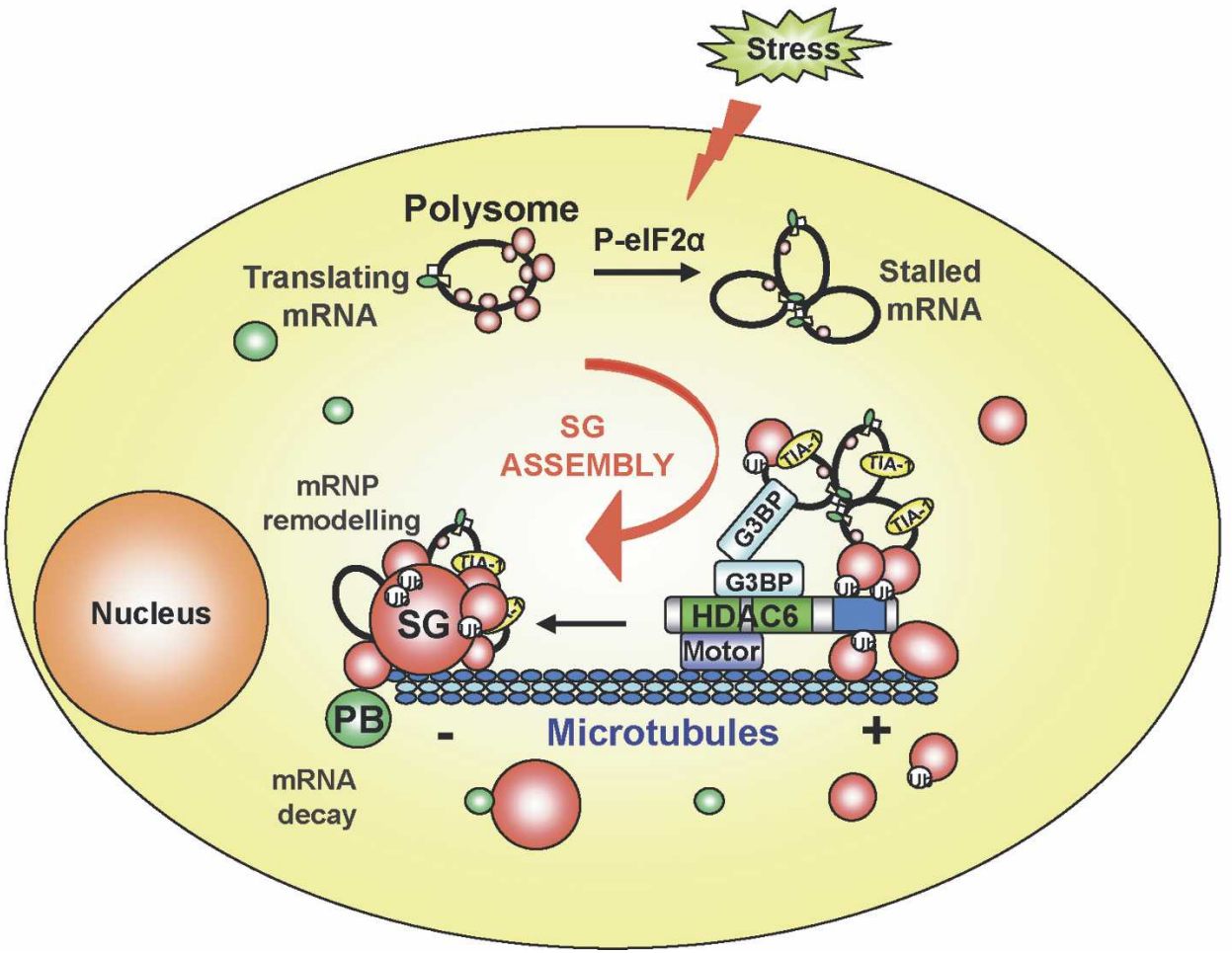

Figure 7. HDAC6 is not required for PB formation. HeLa cells $(A)$ and MEFs $(B)$ (wild-type or $H D A C 6^{-/ y}$ ) were control-treated or treated with $1 \mathrm{mM}$ arsenite for $1 \mathrm{~h}$. Cells were then fixed, immunostained for HDAC6 or G3BP (red) and DCP1a (green), and analyzed by confocal microscopy. The squared insert presents a high magnification in the corner of the merged pictures. Bar, $5 \mu \mathrm{m}$. $(C)$ Model summarizing the findings. Following cellular stress, eIF2 $\alpha$ becomes phosphorylated and leads to stalled polysomes. HDAC6 then nucleates formation of SGs by interacting directly and indirectly with microtubules and motor proteins, G3BP and ubiquitinated proteins that are components of SGs. SGs and their precursors are depicted by red circles of various sizes, some of which are positive for ubiquitin (Ub). PBs are represented by green circles. The blue box in HDAC6 depicts the ZnF-UBP domain that binds to ubiquitin. Some of the SG components may loosely interact with microtubules; see text for details.

teins, in agreement with previous reports. However, we found that under stress conditions the two proteins colocalize in SGs. The presence of HDAC6 in SGs was confirmed in several cell lines (data not shown), as well as under all stress conditions tested. Remarkably, we found that HDAC6 is critical for SG assembly: Both 
pharmacological and genetic inactivation of HDAC6 dramatically impair the formation of SGs, as can be monitored by different markers. For example, TIA-1 has been shown to self-aggregate via prion-like domains and to be important for formation of SGs (Gilks et al. 2004). It is a mostly nuclear protein under normal conditions, which moves into cytoplasmic SGs following stress. However, in cells lacking HDAC6, TIA-1 fails to relocalize and remains largely nuclear. Unlike G3BP, TIA-1 does not appear to interact with HDAC6 (data not shown). We found that the first step of stress-induced translational arrest, the phosphorylation of eIF $2 \alpha$, is not significantly affected by the absence of HDAC6, neither in its magnitude nor in its kinetic of appearance. In agreement with this, global arrest of translation following stress is normal in cells lacking HDAC6. These data demonstrate that HDAC6 controls SG formation downstream from eIF2 $\alpha$ phosphorylation. A recent study has shown that inhibition of ribosome recruitment by eIF4A inhibitors (hippuristanol and pateamine) induces SG formation independently of eIF2 $\alpha$ phosphorylation (Mazroui et al. 2006). We propose that also in this case HDAC6 is required for SG formation, but this remains to be tested. Interestingly, our observations also demonstrate that stress-induced translational repression can take place in absence of visible SGs, as evidenced in HDAC6-deficient cells.

Unlike SGs, PBs are not affected by the chemical inhibition of HDAC activity (data not shown) or by the absence of HDAC6. Although the two structures are often found next to each other, and may be in direct contact-possibly exchanging mRNAs or mRNPs (Kedersha et al. 2005)-PBs are present in normal numbers and shape in cells totally lacking HDAC6. This is also in line with the notion that PBs, where several forms of RNA degradation such as ARE-mediated or nonsense-mediated mRNA decay take place (Sheth and Parker 2006; Franks and Lykke-Andersen 2007), are constantly present in the cell, while SGs are only present (or visible) following stress. Furthermore, PBs are found in yeast, but SGs are not; in this context, it is interesting to note that there is no direct ortholog of HDAC6 in yeast.

\section{Formation of SGs requires intact HDAC6 function}

We showed that intact deacetylase activity of HDAC6 is necessary for facilitating SG assembly, indicating that some protein(s) need to be deacetylated in order for the granules to form efficiently. Whether HSP90, which is deacetylated by HDAC6, may be implicated in this process remains to be tested. In addition, we demonstrated the importance of the microtubules network for SG formation (see below). It is therefore possible that the level of microtubules acetylation, directly controlled by HDAC6, is also important in this process.

Surprisingly, we found that the ZnF-UBP domain of HDAC6, which binds ubiquitin, is also critical: Cells expressing only a non-ubiquitin-binding mutant HDAC6 are even more impaired in the formation of SGs than knockout cells. So far, little is known about the role of ubiquitin or ubiquitinated proteins for SG formation; however, we discovered that SGs can be very efficiently detected by staining for ubiquitin. Thus, SGs also contain ubiquitinated proteins whose interaction with HDAC6 appears to be necessary for their formation. Interestingly, we also found that short-term inhibition of the ubiquitin-proteasome pathway leads to appearance of SGs, in good agreement with very recent data (Mazroui et al. 2007). Furthermore, Roquin, a RING-type ubiquitin ligase required to repress follicular helper $\mathrm{T}$ cells and autoimmunity, also localizes to SGs (Vinuesa et al. 2005). We thus propose that HDAC6 may bind to specific ubiquitinated proteins and recruit them to the SGs, in a manner somewhat analogous to the recruitment of misfolded ubiquitinated proteins into an aggresome (see model in Fig. 7C).

In the case of stress induced by ubiquitinated misfolded proteins, it has been shown that HDAC6 exerts a protective effect for the cell and that the absence of HDAC6 function leads to increased apoptosis (Kawaguchi et al. 2003). We showed here that cells lacking intact HDAC6, and consequently impaired in SG formation, also fail to recover properly from arsenite-induced oxidative stress and undergo apoptosis. This case, as well the protective effect mediated by HDAC6, requires both the deacetylase activity and the ubiquitin-binding function of this enzyme. These observations indicate that HDAC6 plays a general role in the stress response, possibly through its capacity to regulate SGs.

\section{HDAC6 links the microtubule system and SGS}

It is well known that cellular mRNPs can be transported along microtubules by motor proteins. In Xenopus oocytes, the translocation of the veg-1 RNA to the vegetal axis requires intact microtubules (Yisraeli et al. 1990), and in Drosophila oocytes, the plus-end-directed motor protein kinesin I is required for the posterior localization of oskar mRNA and Staufen protein (Micklem et al. 2000). Interestingly, dDcp 1 of the oskar mRNP complex in Drosophila oocytes mislocates in mutants in which microtubule organization is abnormal (Lin et al. 2006). Also, the Argonaute 1 homolog from sea urchins, Seawi, has been identified in a microtubule-associated protein complex that localizes in cytoplasmic puncta (Rodriguez et al. 2005). It is not known whether SGs contain proteins associated with microtubules, but we and others (Ivanov et al. 2003) observed that microtubule integrity is necessary for SG assembly. Furthermore, by using inhibitors of motor proteins, we evidenced here that action of dynein motor proteins is also critical for SG assembly. In addition, we found that SG components such as TIA-1 or eIF3 can be partially coimmunoprecipitated with tubulin, indicating that they might loosely associate with the microtubule network already prior to SG assembly. Collectively, these data suggest that minus-end-directed transport on microtubules is a mechanism used by cells to enhance the efficiency of SGs assembly. Interestingly, it has been previously reported that HDAC6 colocalizes with the p150 15 lued-containing motor complex and con- 
trols microtubule motor-based cargo transport (Hubbert et al. 2002). Because it can bind ubiquitin and also the dynein motor proteins, HDAC6 acts as an adapter, promoting the accumulation of ubiquitinated misfolded proteins in an aggresome (Kawaguchi et al. 2003). As we show here, a similar mechanism may underlie the assembly of SGs (see model in Fig. 7C).

Furthermore, very recent quantitative analysis has revealed that in HeLa cells and mouse ES cells, Argonaute proteins localize into SGs in a microRNA-dependent manner, suggesting that SGs may be relevant for pathways involving microRNA function (Leung et al. 2006). Together, our data suggest that HDAC6 is at the center of an important node integrating several aspects of the stress response.

\section{Materials and methods}

\section{Cell lines and transfections}

All cells were obtained from the American Type Culture Collection and maintained in Dulbecco's Modified Eagle's Medium (DMEM) with $10 \%$ fetal bovine serum and antibiotics under $5 \%$ $\mathrm{CO}_{2}$. Transfections were performed with FuGene6 (Roche) following the manufacturer's protocol or with the calcium phosphate method. Mice with an invalidated HDAC6 gene were generated (Y. Zhang, S.H. Kwon, T. Yamaguchi, F. Cubizolles, S. Rousseau, M. Kneissel, C. Cao, N. Li, H.L. Cheng, K. Chua, et al., unpubl.), and MEFs were isolated from embryonic day 13.5 (E13.5) male embryos. Subsequently, 3T3 cells and rescuants expressing HDAC6 wild type or mutants were established following standard protocols, as described (Zhang et al. 2006; Boyault et al. 2007b). The stable cell line S-HDAC6-293, overexpressing HDAC6, was made by transfection of HEK 293 cells with a CMV-Flag-hHDAC6 vector and selection for neomycin resistance.

\section{Mass spectrometry}

Ten 10-cm dishes of stable HEK 293 cell lines expressing FlaghHDAC6 protein (S-HDAC6-293) were harvested and lysed. Total extracts were analyzed by immunoprecipitation using antiFlag M2 agarose (Sigma); bound proteins were eluted from the beads using $10 \mathrm{mM} 3 \times$ Flag peptide, separated by SDS-PAGE, and stained with coomassie blue staining. The HDAC6-interacting proteins were identified by LC-MS/MS spectrometry.

\section{Preparation of cell extracts and Western blotting analysis}

Cells were rinsed twice with ice-cold PBS and were then extracted with ice-cold NP-40 lysis buffer $(0.5 \%$ NP-40, $50 \mathrm{mM}$ Tris- $\mathrm{HCl}$ at $\mathrm{pH}$ 7.4, $120 \mathrm{mM} \mathrm{NaCl}, 25 \mathrm{mM} \mathrm{NaF}, 25 \mathrm{mM}$ glycerol phosphate, $1 \mathrm{mM}$ EDTA, $5 \mathrm{mM}$ EGTA, Complete protease inhibitor cocktail tablet [Roche]). Cells were collected and centrifuged at $15,000 \mathrm{~g}$ for $15 \mathrm{~min}$ at $4^{\circ} \mathrm{C}$. Protein concentration was measured with a BCA kit (Pierce). Cell lysates containing $50 \mu \mathrm{g}$ of total protein were subjected to SDS-PAGE on $8 \%-12 \%$ slab gels, and proteins were transferred to nitrocellulose membranes. Membranes were blocked for $1 \mathrm{~h}$ in PBS containing $0.1 \%$ Tween-20 and $10 \%(\mathrm{v} / \mathrm{v})$ horse serum and were incubated overnight with the primary antibody. The membranes were then washed with $0.1 \%$ Tween-20/PBS and incubated for $1 \mathrm{~h}$ with an anti-rabbit/mouse secondary antibody coupled to HRP; bound antibodies were detected with the ECL Western blotting analysis system (Amersham Bioscience). Generally, in lanes labeled "input," $1 / 10$ of the material used for the binding reaction was loaded.

\section{Coimmunoprecipitation and pull-down assays}

For coimmunoprecipitation, $500 \mu \mathrm{g}$ of extracts from either NIH3T3 cells or HEK 293T cells were incubated overnight with the primary antibody at $4^{\circ} \mathrm{C}$ with gentle agitation. After this, $25 \mu \mathrm{L}$ of protein A-Sepharose slurry or protein G-agarose slurry were added and samples were incubated for $1 \mathrm{~h}$ at $4^{\circ} \mathrm{C}$ with gentle agitation. Beads were washed three times with NP-40 lysis buffer and subsequently resuspended and boiled in $20 \mu \mathrm{L}$ of loading buffer for SDS-PAGE. For pull-down assays, extracts from HEK 293 cells stably expressing hFlag-HDAC6 were incubated with equal amounts of GST or GST-G3BP for $3 \mathrm{~h}$ at $4^{\circ} \mathrm{C}$. Twenty-five microliters of glutathione-Sepharose slurry were added and the reactions continued for $1 \mathrm{~h}$ with rotation. Pulldowns were electrophoresed on $8 \%$ SDS-PAGE and subjected to anti-Flag Western blots.

\section{Immunofluorescence microscopy and antibodies}

Exponentially growing cells were plated on eight-chamber Labtek slides and incubated overnight. After drug treatment, cells were washed in PHEM buffer (60 mM PIPES at pH 6.9, 25 mM HEPES at $\mathrm{pH} 7.5,10 \mathrm{mM}$ EGTA at $\mathrm{pH} 7.5,4 \mathrm{mM} \mathrm{MgCl}_{2}$ at $\mathrm{pH}$ 6.9) and fixed with $4 \%$ formaldehyde/PBS for $15 \mathrm{~min}$ at room temperature or with methanol for $10 \mathrm{~min}$ at $-20^{\circ} \mathrm{C}$. Slides were then rinsed three times with $0.2 \%$ Triton X-100/PHEM buffer, and cells were permeabilized with $1 \%$ Triton X-100/PHEM buffer for $5 \mathrm{~min}$ at room temperature. Slides were then incubated in blocking solution ( $3 \% \mathrm{BSA} / \mathrm{PBS}$ ) for $1 \mathrm{~h}$ at room temperature for reducing nonspecific binding of the antibody. Incubation with the primary antibodies was carried out for $3 \mathrm{~h}$ at room temperature or overnight at $4^{\circ} \mathrm{C}$, and the slides were then washed three times with $0.2 \%$ Triton X-100/PHEM buffer. Alexa-Fluor anti-mouse and Alexa-Fluor anti-rabbit immunoglobulin antibodies were used as secondary antibodies. After the slides were washed three times with $0.2 \%$ Triton X-100/PHEM buffer and once with deionized water, they were observed under a confocal microscope (Zeiss Axioplan2 LSM 510 Meta). Pictures were deconvolved with the theoretical PSF calculated within the Huygens software (Scientific Volume Imaging, http://www.svi.nl). The following antibodies were used: antiDCP1a (1:100 [IF], kindly provided by W. Filipowicz), anti-eIF2 $\alpha$ (FL-315; 1:500; Santa Cruz Biotechnology), anti-phos-eIF2 $\alpha$ (1:1000; Cell signaling), anti-eIF3 Santa Cruz Biotechnology), anti-Flag M2 (1:2000; Sigma), antiG3BP (1:1000, 1:200 [IF]; BD Transduction Laboratories), antiHA (1:1000; Santa Cruz Biotechnology), anti-HDAC6 (H-300; 1:1000, 1:100 [IF]; Santa Cruz Biotechnology), anti-c-myc (9E10), anti- $\alpha$-tubulin (DM1A; 1:1000; Santa Cruz Biotechnology), anti$\beta$-tubulin (TUB 2.1; 1:1000, 1:200 [IF]; Santa Cruz Biotechnology), anti- $\gamma$-tubulin (GTU-88; 1:1000, 1:200 [IF]; Santa Cruz Biotechnology), anti-Ace-tubulin (TU6-1; 1:1000; Santa Cruz Biotechnology), anti-TIA-1/TIAR (H-120; 1:1000, 1:100 [IF]; Santa Cruz Biotechnology), anti-Ubiquitin (1:100 [IF]; Santa Cruz Biotechnology), or anti-mHDAC-6 (Verdel et al. 2000; 1:1000, 1:100 [IF]). IF refers to the dilutions used for immunofluorescence; other dilutions are for Western analysis.

\section{Quantification of SG-containing cells and SG size}

For quantification, nine fields of each sample were randomly selected. The occurrence of SGs was estimated as the average number of SG-containing cells. For quantification of large SGs, 
100 cells of each sample were randomly selected. The number of large SG was counted using confocal microscopy software (LSM viewer, Carl Zeiss).

\section{Acknowledgments}

We thank Dr. Daniel Hess and Ragna Sack for mass spectrometric analysis; Drs. Patrick Schwarb, Jens Rietdorf, and Aaron Ponti for help with microscopy and image analysis; Dr. Jamal Tazi (IGMM, Montpellier) for the kind gift of a GFP-G3BP construct; Drs. Witold Filipowicz, Yoshikuni Nagamine, Fabien Cubizolles, and Saadi Khochbin (Institut Albert Bonniot, Grenoble) for critical reading of the manuscript; as well as the Matthias laboratory members and Saadi Khochbin for stimulating discussions. This work was supported by the Novartis Research Foundation.

\section{References}

Anderson, P. and Kedersha, N. 2002. Stressful initiations. J. Cell Sci. 115: 3227-3234.

Anderson, P. and Kedersha, N. 2006. RNA granules. J. Cell Biol. 172: 803-808.

Bali, P., Pranpat, M., Bradner, J., Balasis, M., Fiskus, W., Guo, F., Rocha, K., Kumaraswamy, S., Boyapalle, S., Atadja, P., et al. 2005. Inhibition of histone deacetylase 6 acetylates and disrupts the chaperone function of heat shock protein 90: A novel basis for antileukemia activity of histone deacetylase inhibitors. J. Biol. Chem. 280: 26729-26734.

Bertolotti, A., Zhang, Y., Hendershot, L.M., Harding, H.P., and Ron, D. 2000. Dynamic interaction of BiP and ER stress transducers in the unfolded-protein response. Nat. Cell Biol. 2: 326-332.

Bertos, N.R., Gilquin, B., Chan, G.K., Yen, T.J., Khochbin, S., and Yang, X.J. 2004. Role of the tetradecapeptide repeat domain of human histone deacetylase 6 in cytoplasmic retention. J. Biol. Chem. 279: 48246-48254.

Boyault, C., Gilquin, B., Zhang, Y., Rybin, V., Garman, E., Meyer-Klaucke, W., Matthias, P., Muller, C.W., and Khochbin, S. 2006. HDAC6-p97/VCP controlled polyubiquitin chain turnover. EMBO J. 25: 3357-3366.

Boyault, C., Sadoul, K., Pabion, M., and Khochbin, S. 2007a. HDAC6, at the crossroads between cytoskeleton and cell signaling by acetylation and ubiquitination. Oncogene 26: 5468-5476.

Boyault, C., Zhang, Y., Fritah, S., Caron, C., Gilquin, B., Kwon, S.H., Garrido, C., Yao, T.P., Vourc'h, C., Matthias, P., et al. 2007b. HDAC6 controls major cell response pathways to cytotoxic accumulation of protein aggregates. Genes \& Dev. 21: 2172-2181.

Caron, C., Col, E., and Khochbin, S. 2003. The viral control of cellular acetylation signaling. Bioessays 25: 58-65.

Franks, T.M. and Lykke-Andersen, J. 2007. TTP and BRF proteins nucleate processing body formation to silence mRNAs with AU-rich elements. Genes \& Dev. 21: 719-735.

Gallouzi, I.E., Parker, F., Chebli, K., Maurier, F., Labourier, E., Barlat, I., Capony, J.P., Tocque, B., and Tazi, J. 1998. A novel phosphorylation-dependent RNase activity of GAP-SH3 binding protein: A potential link between signal transduction and RNA stability. Mol. Cell. Biol. 18: 3956-3965.

Gilks, N., Kedersha, N., Ayodele, M., Shen, L., Stoecklin, G., Dember, L.M., and Anderson, P. 2004. Stress granule assembly is mediated by prion-like aggregation of TIA-1. Mol. Biol. Cell 15: 5383-5398.

Glozak, M.A., Sengupta, N., Zhang, X., and Seto, E. 2005.
Acetylation and deacetylation of non-histone proteins. Gene 363: $15-23$.

Grozinger, C.M., Hassig, C.A., and Schreiber, S.L. 1999. Three proteins define a class of human histone deacetylases related to yeast Hdalp. Proc. Natl. Acad. Sci. 96: 4868-4873.

Guardiola, A.R. and Yao, T.P. 2002. Molecular cloning and characterization of a novel histone deacetylase HDAC10. J. Biol. Chem. 277: 3350-3356.

Hook, S.S., Orian, A., Cowley, S.M., and Eisenman, R.N. 2002. Histone deacetylase 6 binds polyubiquitin through its zinc finger (PAZ domain) and copurifies with deubiquitinating enzymes. Proc. Nat1. Acad. Sci. 99: 13425-13430.

Hubbert, C., Guardiola, A., Shao, R., Kawaguchi, Y., Ito, A., Nixon, A., Yoshida, M., Wang, X.F., and Yao, T.P. 2002. HDAC6 is a microtubule-associated deacetylase. Nature 417: 455-458.

Irvine, K., Stirling, R., Hume, D., and Kennedy, D. 2004. Rasputin, more promiscuous than ever: A review of G3BP. Int. J. Dev. Biol. 48: 1065-1077.

Ivanov, P.A., Chudinova, E.M., and Nadezhdina, E.S. 2003. Disruption of microtubules inhibits cytoplasmic ribonucleoprotein stress granule formation. Exp. Cell Res. 290: 227-233.

Kawaguchi, Y., Kovacs, J.J., McLaurin, A., Vance, J.M., Ito, A., and Yao, T.P. 2003. The deacetylase HDAC6 regulates aggresome formation and cell viability in response to misfolded protein stress. Cell 115: 727-738.

Kedersha, N. and Anderson, P. 2002. Stress granules: Sites of mRNA triage that regulate mRNA stability and translatability. Biochem. Soc. Trans. 30: 963-969.

Kedersha, N.L., Gupta, M., Li, W., Miller, I., and Anderson, P. 1999. RNA-binding proteins TIA-1 and TIAR link the phosphorylation of eIF- $2 \alpha$ to the assembly of mammalian stress granules. J. Cell Biol. 147: 1431-1442.

Kedersha, N., Cho, M.R., Li, W., Yacono, P.W., Chen, S., Gilks, N., Golan, D.E., and Anderson, P. 2000. Dynamic shuttling of TIA-1 accompanies the recruitment of mRNA to mammalian stress granules. J. Cell Biol. 151: 1257-1268.

Kedersha, N., Chen, S., Gilks, N., Li, W., Miller, I.J., Stahl, J., and Anderson, P. 2002. Evidence that ternary complex (eIF2GTP-tRNA ${ }_{i}^{\text {Met }}$-deficient preinitiation complexes are core constituents of mammalian stress granules. Mol. Biol. Cell 13: $195-210$.

Kedersha, N., Stoecklin, G., Ayodele, M., Yacono, P., LykkeAndersen, J., Fritzler, M.J., Scheuner, D., Kaufman, R.J., Golan, D.E., and Anderson, P. 2005. Stress granules and processing bodies are dynamically linked sites of mRNP remodeling. J. Cell Biol. 169: 871-884.

Kennedy, D., French, J., Guitard, E., Ru, K., Tocque, B., and Mattick, J. 2001. Characterization of G3BPs: Tissue specific expression, chromosomal localisation and rasGAP(120) binding studies. J. Cell. Biochem. 84: 173-187.

Kouzarides, T. 2000. Acetylation: A regulatory modification to rival phosphorylation? EMBO J. 19: 1176-1179.

Kovacs, J.J., Murphy, P.J., Gaillard, S., Zhao, X., Wu, J.T., Nicchitta, C.V., Yoshida, M., Toft, D.O., Pratt, W.B., and Yao, T.P. 2005. HDAC6 regulates Hsp90 acetylation and chaperone-dependent activation of glucocorticoid receptor. Mol. Cell 18: 601-607.

Leung, A.K., Calabrese, J.M., and Sharp, P.A. 2006. Quantitative analysis of Argonaute protein reveals microRNA-dependent localization to stress granules. Proc. Natl. Acad. Sci. 103: 18125-18130.

Lin, M.D., Fan, S.J., Hsu, W.S., and Chou, T.B. 2006. Drosophila decapping protein $1, \mathrm{dDcp} 1$, is a component of the oskar mRNP complex and directs its posterior localization in the oocyte. Dev. Cell 10: 601-613. 
Matsuyama, A., Shimazu, T., Sumida, Y., Saito, A., Yoshimatsu, Y., Seigneurin-Berny, D., Osada, H., Komatsu, Y., Nishino, N., Khochbin, S., et al. 2002. In vivo destabilization of dynamic microtubules by HDAC6-mediated deacetylation. EMBO J. 21: 6820-6831.

Matthias, P., Yoshida, M., and Khochbin, S. 2007. HDAC6: A new cellular stress surveillance factor. Cell Cycle (in press).

Mazroui, R., Sukarieh, R., Bordeleau, M.E., Kaufman, R.J., Northcote, P., Tanaka, J., Gallouzi, I., and Pelletier, J. 2006. Inhibition of ribosome recruitment induces stress granule formation independently of eukaryotic initiation factor $2 \alpha$ phosphorylation. Mol. Biol. Cell 17: 4212-4219.

Mazroui, R., Di Marco, S., Kaufman, R.J., and Gallouzi, I.E. 2007. Inhibition of the ubiquitin-proteasome system induces stress granule formation. Mol. Biol. Cell 18: 2603 2618.

Micklem, D.R., Adams, J., Grunert, S., and St Johnston, D. 2000. Distinct roles of two conserved Staufen domains in oskar mRNA localization and translation. EMBO J. 19: 1366-1377.

Pazman, C., Mayes, C.A., Fanto, M., Haynes, S.R., and Mlodzik, M. 2000. Rasputin, the Drosophila homologue of the RasGAP SH3 binding protein, functions in ras- and Rho-mediated signaling. Development 127: 1715-1725.

Pillai, R.S., Bhattacharyya, S.N., Artus, C.G., Zoller, T., Cougot, N., Basyuk, E., Bertrand, E., and Filipowicz, W. 2005. Inhibition of translational initiation by Let-7 MicroRNA in human cells. Science 309: 1573-1576.

Rodriguez, A.J., Seipel, S.A., Hamill, D.R., Romancino, D.P., M, D.I.C., Suprenant, K.A., and Bonder, E.M. 2005. Seawi-A sea urchin piwi/argonaute family member is a component of MT-RNP complexes. RNA 11: 646-656.

Seigneurin-Berny, D., Verdel, A., Curtet, S., Lemercier, C., Garin, J., Rousseaux, S., and Khochbin, S. 2001. Identification of components of the murine histone deacetylase 6 complex: Link between acetylation and ubiquitination signaling pathways. Mol. Cell. Biol. 21: 8035-8044.

Serrador, J.M., Cabrero, J.R., Sancho, D., Mittelbrunn, M., Urzainqui, A., and Sanchez-Madrid, F. 2004. HDAC6 deacetylase activity links the tubulin cytoskeleton with immune synapse organization. Immunity 20: 417-428.

Sheth, U. and Parker, R. 2003. Decapping and decay of messenger RNA occur in cytoplasmic processing bodies. Science 300: $805-808$.

Sheth, U. and Parker, R. 2006. Targeting of aberrant mRNAs to cytoplasmic processing bodies. Cell 125: 1095-1109.

Stoecklin, G., Stubbs, T., Kedersha, N., Wax, S., Rigby, W.F., Blackwell, T.K., and Anderson, P. 2004. MK2-induced tristetraprolin:14-3-3 complexes prevent stress granule association and ARE-mRNA decay. EMBO J. 23: 1313-1324.

Teixeira, D., Sheth, U., Valencia-Sanchez, M.A., Brengues, M., and Parker, R. 2005. Processing bodies require RNA for assembly and contain nontranslating mRNAs. RNA 11: 371382.

Tourriere, H., Gallouzi, I.E., Chebli, K., Capony, J.P., Mouaikel, J., van der Geer, P., and Tazi, J. 2001. RasGAP-associated endoribonuclease G3Bp: Selective RNA degradation and phosphorylation-dependent localization. Mol. Cell. Biol. 21: 7747-7760.

Tourriere, H., Chebli, K., Zekri, L., Courselaud, B., Blanchard, J.M., Bertrand, E., and Tazi, J. 2003. The RasGAP-associated endoribonuclease G3BP assembles stress granules. I. Cell Biol. 160: 823-831.

van Dijk, E., Cougot, N., Meyer, S., Babajko, S., Wahle, E., and Seraphin, B. 2002. Human Dcp2: A catalytically active mRNA decapping enzyme located in specific cytoplasmic structures. EMBO T. 21: 6915-6924.
Verdel, A., Curtet, S., Brocard, M.P., Rousseaux, S., Lemercier, C., Yoshida, M., and Khochbin, S. 2000. Active maintenance of mHDA2/mHDAC6 histone-deacetylase in the cytoplasm. Curr. Biol. 10: 747-749.

Verdin, E., Dequiedt, F., and Kasler, H.G. 2003. Class II histone deacetylases: Versatile regulators. Trends Genet. 19: 286293.

Vinuesa, C.G., Cook, M.C., Angelucci, C., Athanasopoulos, V., Rui, L., Hill, K.M., Yu, D., Domaschenz, H., Whittle, B., Lambe, T., et al. 2005. A RING-type ubiquitin ligase family member required to repress follicular helper $\mathrm{T}$ cells and autoimmunity. Nature 435: 452-458.

Williams, B.R. 2001. Signal integration via PKR. Sci. STKE 2001: RE2. doi: 10.1126/stke.2001.89.re2.

Yisraeli, J.K., Sokol, S., and Melton, D.A. 1990. A two-step model for the localization of maternal mRNA in Xenopus oocytes: Involvement of microtubules and microfilaments in the translocation and anchoring of Vg1 mRNA. Development 108: 289-298.

Zekri, L., Chebli, K., Tourriere, H., Nielsen, F.C., Hansen, T.V., Rami, A., and Tazi, J. 2005. Control of fetal growth and neonatal survival by the RasGAP-associated endoribonuclease G3BP. Mol. Cell. Biol. 25: 8703-8716.

Zhang, Y., Li, N., Caron, C., Matthias, G., Hess, D., Khochbin, S., and Matthias, P. 2003. HDAC-6 interacts with and deacetylates tubulin and microtubules in vivo. EMBO J. 22: 1168-1179.

Zhang, Y., Gilquin, B., Khochbin, S., and Matthias, P. 2006. Two catalytic domains are required for protein deacetylation. $J$. Biol. Chem. 281: 2401-2404. 


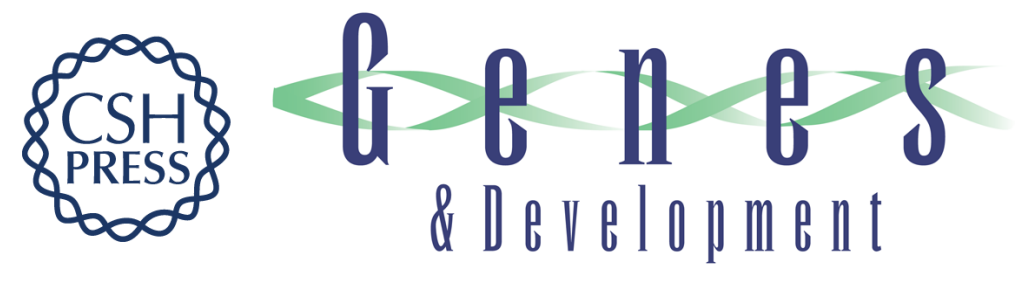

\section{The deacetylase HDAC6 is a novel critical component of stress granules involved in the stress response}

SoHee Kwon, Yu Zhang and Patrick Matthias

Genes Dev. 2007, 21:

Access the most recent version at doi:10.1101/gad.461107

Supplemental http://genesdev.cshlp.org/content/suppl/2007/11/28/21.24.3381.DC1
Material

References This article cites 54 articles, 35 of which can be accessed free at: http://genesdev.cshlp.org/content/21/24/3381.full.html\#ref-list-1

License

Email Alerting Receive free email alerts when new articles cite this article - sign up in the box at the top Service right corner of the article or click here.

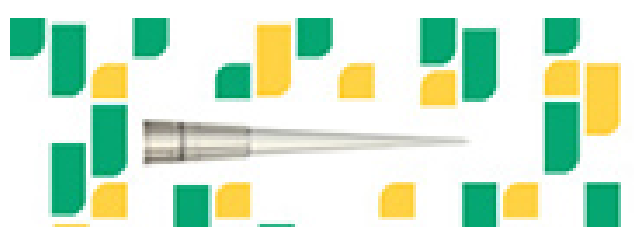

